\title{
MODELAGEM DE CARGAS VARIANTES NO TEMPO NA AVALIAÇÃO DE CONFIABILIDADE COMPOSTA VIA SIMULAÇÃO MONTE CARLO NÃO-SEQÜENCIAL
}

\author{
Luiz A. F. Manso* \\ Armando M. Leite da Silva ${ }^{\dagger}$ \\ manso@mgconecta.com.br \\ armando@iee.efei.br \\ *Departamento de Engenharia Elétrica - DEPEL, Universidade Federal de São João del-Rei - UFSJ. \\ ${ }^{\dagger}$ Grupo de Engenharia de Sistemas - GESis, Universidade Federal de Itajubá - UNIFEI.
}

\begin{abstract}
This paper presents a new methodology to evaluate the reliability of composite generation and transmission systems considering time varying loads. Based on non-sequential Monte Carlo simulation, the proposed method uses a special Markov load model to represent different chronological load patterns per area or bus. A new estimating process, named one step forward state transition, is proposed to evaluate LOLF (loss of load frequency) index, without assuming any coherent behavior in the system. Case studies using the MRTS (Modified IEEE Reliability Test System) and the SSB (Brazilian South-Southeastern System) are presented and discussed.
\end{abstract}

KEYWORDS: Composite reliability, Monte Carlo simulation, generation and transmission reliability, uncertainty modeling.

\section{RESUMO}

Este artigo apresenta uma nova metodologia para avaliar a confiabilidade de sistemas compostos de geração e transmissão considerando cargas variantes no tempo. Baseado na simulação Monte Carlo não-seqüencial, o método proposto utiliza um modelo de Markov capaz

Artigo submetido em $14 / 12 / 02$

1a. Revisão em 10/04/03; 2a. Revisão em 04/08/03

Aceito sob recomendação do Ed. Assoc. Prof. Takashi Yoneyama de representar diferentes padrões cronológicos de carga para cada área ou barra do sistema. Um novo processo de estimação é proposto para avaliar o índice de freqüência de corte de carga (LOLF - loss of load frequency), sem a necessidade de presumir comportamento coerente para o sistema. Exemplos utilizando os sistemas MRTS (Modified IEEE Reliability Test System) e SSB (SulSudeste Brasileiro) são apresentados e discutidos.

PALAVRAS-CHAVE: Confiabilidade composta, simulação Monte Carlo, confiabilidade da geração e transmissão, modelagem de incertezas.

\section{INTRODUÇÃO}

Para sistemas de geração e transmissão as estimativas dos índices de perda de carga são obtidas através de algoritmos de confiabilidade, os quais são baseados em duas representações distintas: espaço de estados e cronológica. Em geral, os algoritmos baseados na representação a espaço de estados são compostos de três passos principais (EPRI, 1982):

(i) selecione um estado do sistema $x^{k}$ (i.e. nível de carga, disponibilidade dos equipamentos, etc.);

(ii) analise o desempenho do estado selecionado (i.e. verifique se a configuração de geradores e circuitos é capaz de atender à carga definida para este estado sem violar limites operativos; se necessário, acione 
medidas corretivas tais como redespacho de geração, correção de tensões, corte de carga, etc.);

(iii) estime índices de confiabilidade; se a precisão dos estimadores é aceitável, pare; senão, retorne ao passo (i).

A enumeração de estados e a simulação Monte Carlo não-seqüencial são exemplos de algoritmos baseados na representação a espaço de estados, a qual utiliza modelos de Markov para reproduzir as transições de estado de equipamentos e da carga. Portanto, os estados são selecionados e avaliados sem qualquer conexão cronológica ou memória (Pereira e Balu, 1992).

Os passos necessários à avaliação de índices de confiabilidade considerando a representação cronológica (simulação Monte Carlo seqüencial) são, a princípio, os mesmos utilizados pela representação a espaço de estados (Salvaderi, 1990). A diferença básica está na forma como são gerados os estados do sistema, i.e. passo (i) do algoritmo. No caso cronológico, os estados são selecionados seqüencialmente no tempo, o que permite representar aspectos dependentes do tempo, como diferentes padrões cronológicos de carga por área ou barra do sistema. Entretanto, na modelagem cronológica dois estados consecutivos do sistema diferem um do outro apenas pelo estado de um de seus componentes, o que requer um esforço computacional substancialmente maior que o necessário para as demais técnicas.

Uma variável dentre as mais importantes para serem consideradas na avaliação da confiabilidade é, seguramente, o padrão cronológico da carga (Melo et alii, 1993). A carga do sistema é usualmente representada por modelos de Markov, com grande agregação de seus estados (Breipohl et alii, 1992; Melo et alii, 1993; Mello et alii, 1997 a; Manso et alii, 1999). Estes modelos assumem correlação 1 entre as cargas das barras e a carga total do sistema, o que pode não ser válido para diversos sistemas (Wenyuan e Billinton, 1991). Considere, por exemplo, um sistema interligado o qual atende a diferentes regiões. O comportamento da carga em cada região é determinado por características regionais, tais como composição da carga, condições climáticas, programas de gerenciamento da demanda, etc. Além disso, a participação das diversas classes consumidoras (e.g. residencial, comercial, industrial, etc.) é geralmente diferente em cada barra do sistema. A combinação destes fatores produz padrões regionais distintos para o comportamento da carga (Sankarakrishnan e Billinton, 1995).
Para sistemas de potência de grande porte, os métodos de avaliação da confiabilidade baseados em simulação Monte Carlo são mais atrativos que os métodos de enumeração de estados (Pereira e Balu, 1992; Salvaderi, 1990; Melo et alii, 1993). Dentre os métodos de simulação, três opções têm sido consideradas: nãoseqüencial, seqüencial e pseudo-seqüencial. Os métodos não-seqüencial e pseudo-seqüencial Markoviano (Melo et alii, 1993; Mello et alii, 1997 a; Manso et alii, 1999), por serem baseados em modelos de Markov agregados para a representação da carga, não são hábeis para considerar cargas variantes no tempo (Mello et alii, 1997 b), i.e. diferentes padrões de carga por área ou barra do sistema. A simulação Monte Carlo seqüencial é uma ferramenta natural para simular aspectos cronológicos. Entretanto, a modelagem cronológica requer esforço computacional substancialmente maior que aquele apresentado pelas técnicas não-seqüencial e pseudo-seqüencial Markoviana (Singh et alii, 1993; Manso et alii, 1999). Uma outra simulação pseudo-seqüencial (Mello et alii, 1994) mantém a flexibilidade e a precisão da simulação Monte Carlo seqüencial, porém o speed-up obtido não atinge a mesma eficiência da simulação não-seqüencial.

Recentemente foi proposto um novo método (Leite da Silva et alii, 2000), denominado simulação Monte Carlo pseudo-cronológica, o qual retém a eficiência computacional da simulação não-seqüencial e a habilidade para representar curvas cronológicas de carga da simulação seqüencial. Este método utiliza um modelo de Markov para a carga, o qual foi concebido para capturar o impacto das cargas variantes no tempo. A implementação deste método em algoritmos de simulação Monte Carlo não-seqüencial pré-existentes, embora viável, requer um considerável esforço de programação.

A partir do modelo de Markov proposto por (Leite da Silva et alii, 2000), este artigo apresenta uma nova metodologia para avaliar a confiabilidade de sistemas compostos de geração e transmissão considerando diferentes padrões de carga por área ou barra do sistema. Baseado na simulação Monte Carlo não-seqüencial, o método proposto utiliza um novo processo de estimação, chamado de transição de estado um passo à frente, para avaliar o índice LOLF (loss of load frequency) sem a necessidade de presumir comportamento coerente para o sistema. Esta hipótese é adotada por dois eficientes métodos (Melo et alii 1992) e (Melo et alii 1993). As diferenças entre estas metodologias e a técnica proposta são amplamente discutidas e ilustradas através de diferentes sistemas testes. 


\section{METODOLOGIA PROPOSTA}

\subsection{Modelo de Markov com múltiplos ní- veis}

Qualquer modelo de carga irá reproduzir de maneira aproximada o seu real comportamento. A precisão de cada modelo depende da quantidade e qualidade de dados disponíveis. Modelos de Markov podem ser utilizados não apenas para reproduzir o processo de falha e reparo de equipamentos, mas também para representar o comportamento da carga.

Usualmente curvas cronológicas da carga com 8760 pontos horários são disponíveis e fornecem informações extremamente úteis aos estudos de confiabilidade de sistemas de potência (Melo et alii, 1993). Utilizando as hipóteses de Markov, as curvas horárias da carga são transformadas em modelos multi-estados, não balanceados em freqüência. Para reduzir o número de estados a serem analisados, são utilizadas técnicas de agrupamento (clustering techniques) (Anderberg, 1973). Este processo de agregação produz modelos de Markov incapazes de representar cargas variantes no tempo.

Se os componentes do sistema são não-Markovianos e se cargas variantes no tempo são especificadas por área ou barra, ou até mesmo por classe consumidora, a única opção para avaliar índices de confiabilidade é através da simulação Monte Carlo seqüencial ou cronológica. O preço a ser pago por adotar uma representação tão detalhada é um tempo de processamento extremamente elevado. Em se tratando de sistemas de grande porte, esta avaliação pode se tornar inviável. Tendo em mente esta restrição, e considerando que as cargas podem variar no tempo segundo padrões identificados para cada área ou barra, um modelo de Markov não-agregado com múltiplos níveis foi proposto por (Leite da Silva et alii, 2000) para a representação da carga. Este modelo é composto por um conjunto de $T$ estados com níveis múltiplos de carga, conectados na mesma ordem em que aparecem no histórico da carga. Como exemplo, um modelo com 8736 (52 semanas) estados de múltiplos níveis deve ser utilizado, se curvas horárias anuais são consideradas.

Com o objetivo de explicar o modelo, a Figura 1 apresenta um estado amostrado $h$ e o próximo estado $h+1$. Nesta figura, $\mathrm{L}_{h}\left(\mathrm{~A}_{m}\right)$ representa o nível de carga do estado $h$ na área $m$, e $\lambda_{L}$ é a taxa de transição da carga. Como pode ser observado, quando a carga da área 1 transita do estado $h$ para o estado $h+1$, i.e. de $\mathrm{L}_{h}\left(\mathrm{~A}_{1}\right)$ para $\mathrm{L}_{h+1}\left(\mathrm{~A}_{1}\right)$, o mesmo ocorre para as demais áreas, ou seja, $\mathrm{L}_{h}\left(\mathrm{~A}_{2}\right)$ transita para $\mathrm{L}_{h+1}\left(\mathrm{~A}_{2}\right), \ldots$ e $\mathrm{L}_{h}\left(\mathrm{~A}_{m}\right)$ transita para $\mathrm{L}_{h+1}\left(\mathrm{~A}_{m}\right)$. Note que, para uma dada tran-

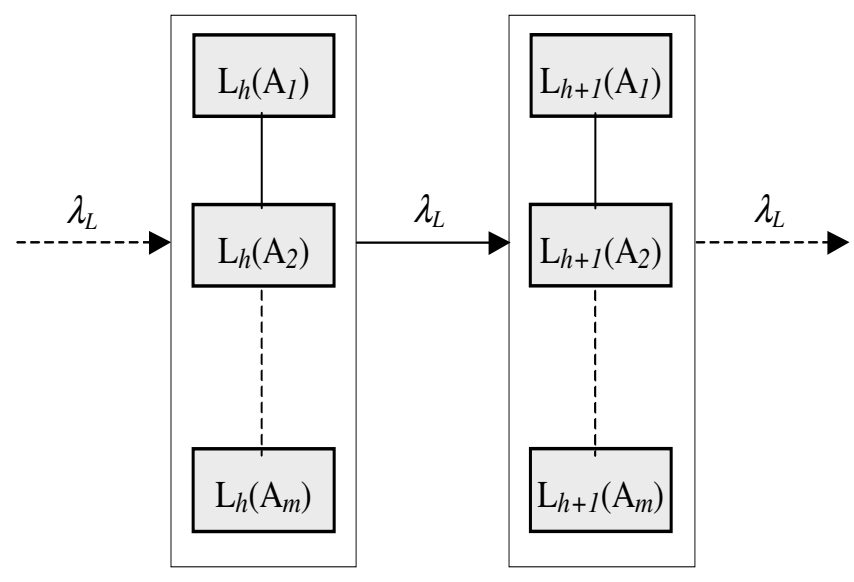

Figura 1: Estados de Carga com Múltiplos Níveis: Amostrado h e Próximo Estado h+1.

sição de estado, o nível de carga pode se elevar em uma ou mais áreas e decair nas demais. Obviamente, o conceito de área pode ser estendido para barra ou classe consumidora.

O modelo a múltiplos níveis da Figura 1 é extremamente flexível e superior à maioria dos modelos de Markov discutidos pela literatura. Uma grande vantagem deste modelo está em manter, de maneira aproximada, a representação cronológica. Em média, o estado $h$ do modelo corresponderá à hora $h$ da curva cronológica da carga (Leite da Silva et alii, 2000). Além disso, a avaliação de índices de confiabilidade composta via simulação nãoseqüencial fica mais flexível: não é mais necessário assumir coerência para o comportamento do sistema, assim como é possível considerar cargas variantes no tempo.

\subsection{Processo de estimação da LOLF}

A simulação não-seqüencial pode fornecer estimativas não-tendenciosas para os índices LOLF e LOLD (loss of load duration). A função teste utilizada para estimar a LOLF $\left(F_{L O L F}\right)$ é dada por (Melo et alii, 1992):

$$
F_{L O L F}\left(x^{k}\right)= \begin{cases}0 & \text { se } x^{k} \in X_{S} \\ \Delta \lambda_{k} & \text { se } x^{k} \in X_{F}\end{cases}
$$

onde $X_{S}$ é o conjunto dos estados de sucesso, $X_{F}$ é o conjunto dos estados de falha e $\Delta \lambda_{k}$ é a soma das taxas de transição do estado de falha $x^{k}$ para todos os estados de sucesso que podem ser alcançados mediante uma transição. A LOLD é obtida por:

$$
L O L D=L O L P / L O L F
$$

onde LOLP (loss of load probability) é a probabilidade de falha. 
A princípio, para cada estado de falha $x^{k} \in \mathrm{X}_{F}$ ) selecionado, com $n_{c}$ componentes, ao menos $n_{c}$ análises adicionais de adequação (i.e. fluxo de potência, medidas corretivas, etc.) serão necessárias para atualizar a estimativa da freqüência, o que resulta em um elevado esforço computacional. Uma técnica para reduzir este esforço computacional utiliza filtros baseados nos multiplicadores de Lagrange produzidos pelo algoritmo de otimização das medidas corretivas (Melo et alii, 1992). No entanto, para que este método possa ser empregado é necessário adotar a hipótese de coerência para o comportamento do sistema:

Se um componente falho é reparado, ou se há redução da carga, o desempenho do sistema nunca piora, e, inversamente, se um componente deixa de operar, ou se há elevação da carga, o desempenho do sistema nunca melhora.

Um outro método, ainda mais eficiente, é baseado no conceito de probabilidade condicionada ou freqüência incremental (Melo et alii, 1993), o que permite calcular $\Delta \lambda_{k}$ utilizando apenas probabilidades e taxas de transição. Este método se mostrou extremamente eficiente, do ponto de vista computacional, e hábil para lidar com as transições da carga, as quais exercem papel preponderante na avaliação da LOLF. Entretanto, ele apresenta duas restrições: (i) o uso da hipótese de coerência, e (ii) o uso de um só modelo de Markov para todas as cargas do sistema, o que impõe o mesmo padrão de variação para todas as barras de carga do sistema (coeficiente de correlação igual a 1).

O processo proposto neste artigo para a estimação do índice LOLF é uma técnica extremamente eficiente do ponto de vista computacional. Ao mesmo tempo, ele é flexível o bastante para prescindir da hipótese de coerência e, consequentemente, viabilizar a consideração de cargas variantes no tempo utilizando técnicas nãoseqüenciais. Para isto, é proposta uma nova função teste, dada pela Eq. (3):

$$
F_{L O L F}\left(x^{k}\right)= \begin{cases}0 & \text { se } x^{k} \in X_{S} \\ \lambda_{k}^{\text {out }} & \text { se } x^{k} \in X_{F} \text { e } x^{m} \in X_{S}\end{cases}
$$

onde $\lambda_{k}^{\text {out }}$ é o somatório das taxas de transição do estado de falha $x^{k}$ para todos os estados diretamente ligados a ele, e $x^{m}$ é um estado qualquer, encontrado a partir de $x^{k}$ através de uma simples transição. Conforme será demonstrado, o processo de estimação proposto deve simular somente uma das possíveis transições à frente, partindo do estado de falha $x^{k}$, de modo a selecionar um estado vizinho $x^{m}$. Por esta razão ele é denominado de processo de transição de estado um passo à frente.

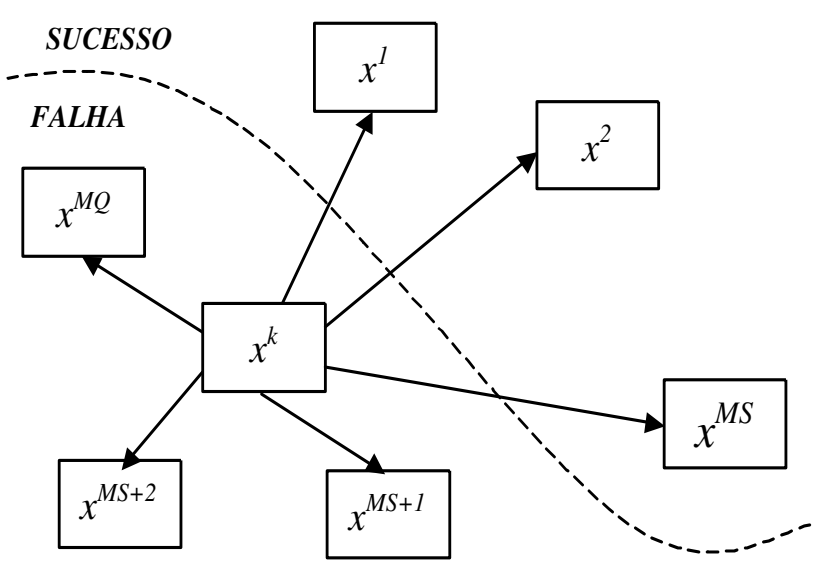

Figura 2: Estado de Falha $x^{k}$ e sua Vizinhança

A Figura 2 é utilizada para explicar o processo de estimação da LOLF. Nesta figura $M Q$ e $M S$ representam, respectivamente, o conjunto de estados quaisquer e o conjunto de estados de sucesso para os quais o sistema pode ir após deixar o estado $x^{k}$.

Este processo de estimação deve primeiramente identificar uma possível transição partindo do estado amostral de falha $x^{k}$ em direção a um estado vizinho $x^{m}$. A freqüência com a qual o sistema transita do estado $x^{k}$ para o estado $x^{m}$, onde a única diferença entre estes dois estados está na alteração do ponto de operação de um componente $j$ (e.g. uma mudança do estado $x_{j}=a$ para o estado $x_{j}=b$, com uma taxa de transição $\lambda_{a b}$ ou $\left.\lambda_{k m}\right)$, é dada por:

$$
f_{k m}=P\left(x^{k}\right) \times \lambda_{a b}=P\left(x^{k}\right) \times \lambda_{k m}
$$

A freqüência de saída do estado $x^{k}$ é dada por:

$$
f_{k}^{\text {out }}=P\left(x^{k}\right) \times \lambda_{k}^{\text {out }}
$$

A probabilidade de ocorrência da transição $\mathrm{km}$, definida como a probabilidade do sistema ingressar no estado $x^{m}$ partindo do estado $x^{k}$, é avaliada como a razão entre as freqüências $f_{k m}$ e $f_{k}^{o u t}$, i.e.:

$$
P_{k m}=f_{k m} / f_{k}^{\text {out }}=\lambda_{k m} / \lambda_{k}^{\text {out }}=\lambda_{k m} / \sum_{i=1}^{M Q} \lambda_{k i}
$$

onde $M Q$ representa o conjunto de estados quaisquer para os quais o sistema pode ingressar após deixar o estado $x^{k}$, conforme definido na Figura 2.

A Eq. (6) fornece a base para a construção da função distribuição de probabilidade associada com as possíveis 
transições de partida do estado amostral de falha $x^{k}$. Através desta função de distribuição um estado vizinho $x^{m}$ pode ser amostrado e a nova função teste para a LOLF, dada pela Eq. (3), pode ser empregada.

Considerando que a taxa de transição do estado $x^{k}$ para o estado $x^{m}$ é:

$$
\lambda_{k m}=P_{k m} \times \lambda_{k}^{\text {out }}
$$

pode-se afirmar que a soma das taxas de transição entre o estado de falha $x^{k}$ e todos os estados de sucesso, os quais podem ser alcançados a partir de $x^{k}$ através de uma simples transição $\left(\Delta \lambda_{k}\right.$ na Eq. (1)), é dada por:

$$
\Delta \lambda_{k}=\sum_{i=1}^{M S} \lambda_{k i}=\sum_{i=1}^{M S}\left(P_{k i} \times \lambda_{k}^{\text {out }}\right)=\left(\sum_{i=1}^{M S} P_{k i}\right) \times \lambda_{k}^{\text {out }}
$$

onde $M S$ representa o conjunto de estados de sucesso para os quais o sistema pode ingressar após deixar o estado de falha $x^{k}$, conforme definido pela Figura 2.

Considerando, ainda, que o somatório $\sum_{i=1}^{M S} P_{k i}$ é naturalmente obtido pela combinação do processo de estimação proposto com a simulação não-seqüencial, é possível estabelecer que a função teste (3) está em completa conformidade com a função teste (1).

Note que é necessário simular somente uma transição partindo de cada estado de falha $x^{k}$. Portanto, o número de análises adicionais de desempenho é pequeno, e consequentemente, o acréscimo do esforço computacional é extremamente baixo. Este acréscimo pode ser expresso em termos do número de análises adicionais de adequação, o qual é dado pelo produto $L O L P \times N S$, onde $N S$ é o número de sorteios originais da simulação não-seqüencial.

\section{3 Índices de Probabilidade e Energia}

As funções teste utilizadas para estimar os índices LOLP - loss of load probability e EENS - expected energy not supplied dependem somente da função distribuição de probabilidade associada aos estados do sistema $x^{k}$, a qual por sua vez depende das distribuições de probabilidade para geradores, equipamentos de transmissão e carga (Leite da Silva et alii, 2000).

Portanto, se a carga está representada pelo modelo de Markov não-agregado com múltiplos níveis, a simulação não-seqüencial pode fornecer estimativas nãotendenciosas para os índices LOLP e EENS quando as chamadas cargas variantes no tempo são consideradas, sem que haja acréscimos no esforço computacional.

\section{EXEMPLOS}

A aplicação do método proposto e da nova função teste para a estimação da LOLF é ilustrada através de estudos de caso utilizando os sistemas MRTS - Modified IEEE Reliability Test System e SSB - Sul-Sudeste Brasileiro. A análise de adequação de cada estado amostrado é realizada através de um fluxo de potência DC e de um algoritmo de otimização das medidas corretivas baseado em programação linear, cujo objetivo é minimizar a quantidade de energia interrompida. Um algoritmo de simulação Monte Carlo seqüencial é utilizado para fornecer estimativas para os índices de confiabilidade. Estas estimativas são adotadas como referência para a comparação dos resultados obtidos através dos modelos de simulação Monte Carlo não-seqüencial: proposto e tradicional (Melo et alii, 1993). Em todos os testes realizados, o critério de parada adotado corresponde à obtenção de um coeficiente de incerteza relativa (Melo et alii, 1992; Pereira e Balu, 1992), para o índice LOLF do sistema $\left(\beta_{L O L F}\right)$ igual a $2 \%$. Um computador Pentium $1.2 \mathrm{GHz}$ foi utilizado em todas as simulações realizadas.

\subsection{Sistema MRTS}

O sistema MRTS resulta de modificações feitas no IEEERTS (IEEE, 1979), com o objetivo de estressar a rede de transmissão. Para tal, são duplicadas a capacidade de geração e a carga, em cada barra do sistema. A nova capacidade instalada atinge a $6810 \mathrm{MW}$ e o pico de carga fica igual a 5700 MW. O sistema possui 2 áreas (230 e $138 \mathrm{kV}), 24$ barras, 38 circuitos e 14 usinas geradoras. A curva de carga adotada para a área de $138 \mathrm{kV}$ corresponde a 52 repetições da semana pico de inverno ( semana 51 da curva original). Para a área de $230 \mathrm{kV}$ a curva utilizada foi obtida através de 52 repetições da semana 25 da curva original. Estas curvas (168 horas), assim como a curva resultante para o sistema, são apresentadas pela Figura 3.

A Tabela 1 apresenta os índices de confiabilidade estimados através das simulações Monte Carlo seqüencial, não-seqüencial proposta (NSeq-Prop) e não-seqüencial tradicional (NSeq-Trad), a qual utiliza as técnicas propostas por (Melo et alii, 1993). Nas simulações realizadas a curva de carga resultante do sistema, dada pela Figura 3, é adotada para representar o padrão da carga em todas as barras. Neste caso, os dois métodos nãoseqüenciais utilizaram um modelo de Markov com nível único, obtido a partir da curva de carga resultante do sistema.

A simulação seqüencial analisou 3.853 .369 casos, distribuídos em 454 anos, e necessitou de 18,1 minutos 


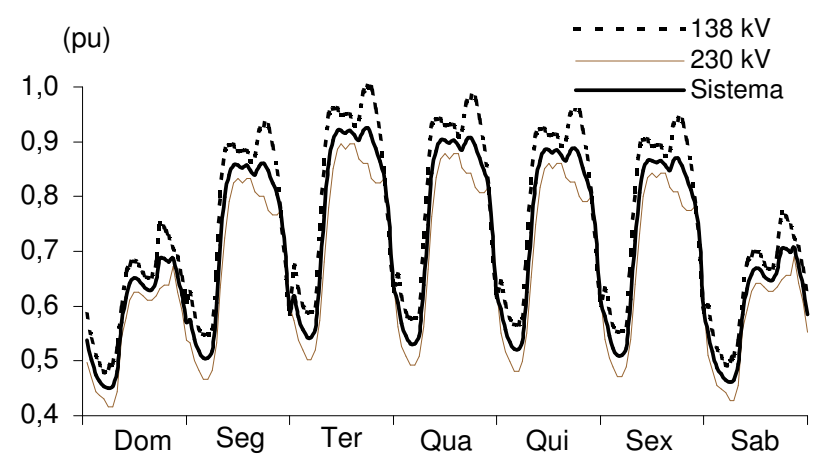

Figura 3: Curvas Semanais de Carga - MRTS

Tabela 1: Índices Estimados para o MRTS - Curva de Carga do Sistema

\begin{tabular}{|c|c|c|c|}
\hline Indice & Seqüencial & NSeq-Prop & NSeq-Trad \\
\hline LOLP $\left(\times 10^{2}\right)$ & 1,8460 & 1,8955 & 1,8877 \\
\hline EENS(GWh/a) & 24,397 & 24,591 & 24,701 \\
\hline LOLF(oc./a) & 28,493 & 30,344 & 30,330 \\
\hline LOLD(h) & 5,6598 & 5,4721 & 5,4521 \\
\hline
\end{tabular}

para realizar os cálculos. Durante a simulação nãoseqüencial proposta foram analisados 769.957 casos (incluindo 14.323 casos adicionais) em um tempo total de processamento de 3,2 minutos. Já a simulação nãoseqüencial tradicional analisou 1.638 .432 casos, necessitando de 6,9 minutos para o processamento dos cálculos.

Neste caso apenas uma curva de carga (a curva resultante para o sistema) é utilizada. Portanto, o desempenho dos métodos, em termos de precisão dos índices estimados, é muito similar. Considerando o sistema MRTS, o método proposto se mostrou mais veloz que o tradicional. Para obter um mesmo coeficiente de incerteza relativa (2\%) para o índice LOLF do sistema, a técnica proposta apresentou um speed up de 2,15 em relação ao método tradicional.

Analogamente à Tabela 1, a Tabela 2 mostra os índices de confiabilidade estimados quando as curvas de carga $138 \mathrm{kV}$ e $230 \mathrm{kV}$, dadas pela Figura 3, são utilizadas para representar o padrão das cargas em suas respectivas áreas. O método não-seqüencial proposto utilizou um modelo de Markov não-agregado com múltiplos niveis para reproduzir o comportamento da carga descrito por estas duas curvas. O método não-seqüencial tradicional não é capaz de considerar cargas variantes no tempo. Portanto, os resultados apresentados para este método são exatamente os mesmos obtidos pela análise
Tabela 2: Índices Estimados para o MRTS - Curvas de Carga por Área

\begin{tabular}{|c|c|c|c|}
\hline Indice & Seqüencial & NSeq-Prop & NSeq-Trad \\
\hline LOLP $\left(\times 10^{2}\right)$ & 3,3466 & 3,4110 & 1,8877 \\
\hline EENS(GWh/a) & 26,887 & 28,343 & 24,701 \\
\hline LOLF(oc./a) & 54,517 & 55,343 & 30,330 \\
\hline LOLD(h) & 5,3627 & 5,3991 & 5,4521 \\
\hline
\end{tabular}

anterior, quando um modelo de Markov com nível único foi utilizado para a representação da carga.

A simulação seqüencial considerou 171 anos, analisando 1.621.931 casos e utilizando um tempo de 7,4 minutos para realizar os cálculos. A simulação não-seqüencial proposta analisou um total de 440.657 casos (incluindo 14.535 casos adicionais) durante um tempo de processamento de 2,0 minutos. Para a simulação não-seqüencial tradicional o número de casos e o tempo de processamento são os mesmos da análise anterior.

Como pode ser observado, índices de confiabilidade obtidos pela técnica proposta são novamente muito próximos daqueles apresentados pela simulação seqüencial. Contrariamente, o método não-seqüencial tradicional apresenta índices inadequados. Ao considerar a curva de carga resultante para o sistema este método não identifica as variações distintas apresentadas pelas cargas de cada área do sistema. Em relação à freqüência de falha do sistema, o erro relativo apresentado para o índice LOLF estimado pelo método proposto ficou em 1,5\%. Para este mesmo índice o erro cometido pelo método tradicional (Melo et alii, 1993) foi de 42,8\%.

\subsection{Sistema Sul-Sudeste Brasileiro - SSB}

O sistema SSB é composto por 413 barras, 255 unidades geradoras e 685 circuitos. A capacidade instalada e o pico de carga são aproximadamente iguais a 46 GW e 41 GW, respectivamente. A Figura 4 apresenta algumas informações básicas através de um diagrama simplificado contendo a carga pico $(L)$ e capacidade instalada $(G)$ de suas áreas principais.

A Figura 5 apresenta curvas típicas diárias para o mês de junho, para as áreas Minas e Sul. O comportamento distinto da carga é devido à diferente composição da mesma para estas duas áreas. Um outro fator determinante são as condições mais severas presentes na região Sul durante o inverno.

A Figura 6 apresenta curvas de carga típicas semanais para a área Minas e para o sistema. Observe que o pico de carga para o sistema ocorre às quintas-feiras, 


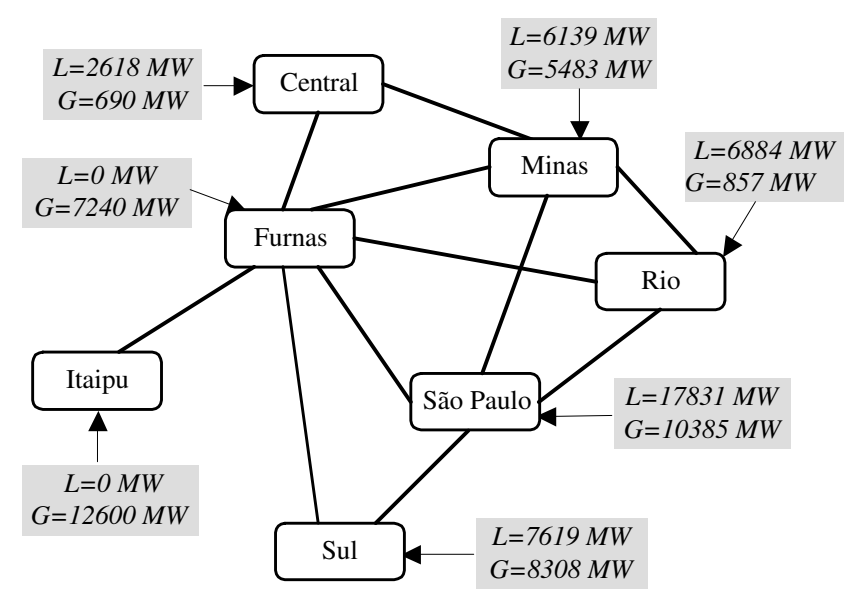

Figura 4: Diagrama Simplificado do SSB.

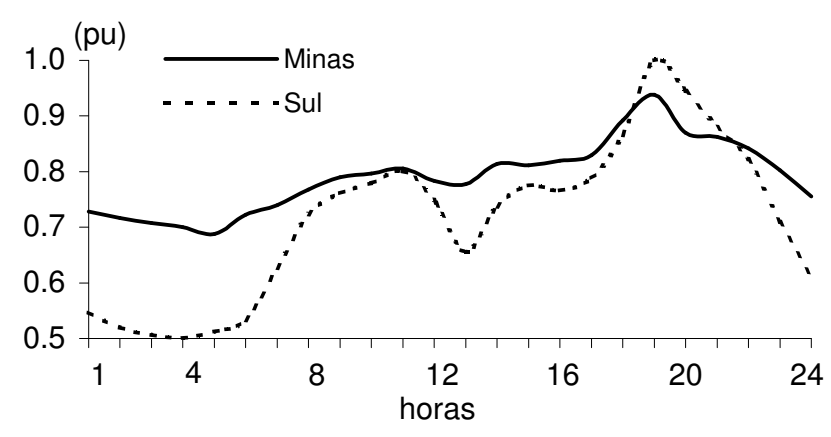

Figura 5: Curvas Diárias de Carga - SSB

enquanto que o pico da área Minas ocorre aos sábados, como conseqüência de eficientes programas de gerenciamento da demanda implementados pela CEMIG. A variedade de padrões de carga ilustra a importância de uma detalhada representação para a cronologia da carga ou, ao menos, de um modelo de carga a espaço de estados, hábil para considerar os diferentes padrões da carga.

A Tabela 3 apresenta os índices de confiabilidade estimados para o sistema SSB, através das simulações Monte Carlo seqüencial, não-seqüencial proposta (NSeq-Prop) e não-seqüencial tradicional (NSeq-Trad). Para representar o comportamento da carga nas cinco áreas: São Paulo, Minas, Rio, Sul e Centro, os métodos seqüencial e não-seqüencial proposto consideraram 5 curvas distintas de carga com 8736 pontos horários. Já a simulação não-seqüencial tradicional utilizou um modelo de Markov com nível único, obtido a partir da curva de carga resultante do sistema, apresentada pela Figura 6 .

A simulação seqüencial considerou 225.935 casos, dis-

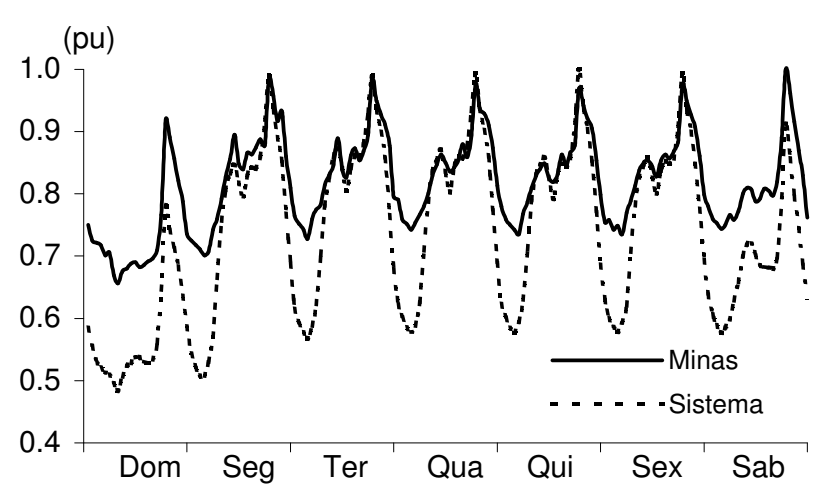

Figura 6: Curvas Semanais de Carga - SSB

Tabela 3: Índices Estimados para o SSB

\begin{tabular}{|c|c|c|c|}
\hline Indice & Seqüencial & NSeq-Prop & NSeq-Trad \\
\hline LOLP $\left(\times 10^{2}\right)$ & 4,5792 & 4,5970 & 3,8681 \\
\hline EENS(GWh/a) & 27,200 & 25,427 & 17,432 \\
\hline LOLF(oc./a) & 152,65 & 154,69 & 105,76 \\
\hline LOLD(h) & 2,6207 & 2,6033 & 3,2039 \\
\hline
\end{tabular}

tribuídos em 17 anos, e necessitou de 5,8 minutos para realizar os cálculos. Durante a simulação nãoseqüencial proposta foram analisados 263.415 casos (incluindo 11.577 casos adicionais) em um tempo total de processamento de 6,6 minutos. Já a simulação nãoseqüencial tradicional analisou 352.136 casos, utilizando 8,9 minutos para o processamento dos cálculos.

Mais uma vez o desempenho do método proposto é excelente quando comparado ao método tradicional. O erro relativo para a estimativa da LOLF obtida pela técnica proposta ficou em 1,3\%, bem abaixo do erro de $31,0 \%$ apresentado pela metodologia tradicional.

Quanto aos tempos de processamento obtidos, que no caso do sistema SSB apresentou uma análise mais rápida para a simulação seqüencial, faz-se necessário o seguinte comentário. Em relação aos demais índices, a convergência do índice LOLF é mais lenta quando se utiliza a simulação não-seqüencial e mais rápida se a simulação seqüencial é utilizada. Este comportamento se torna mais intenso quanto maior for a freqüência de falha do sistema. Para o sistema SSB a incerteza obtida pela estimativa do índice EENS foi de 1,5\% para a simulação não-seqüencial proposta e de $10,72 \%$ para a simulação seqüencial. Para que se tenha a mesma confiança (i.e. $\beta=1,5 \%$ ) para a estimativa da EENS obtida pela simulação seqüencial, o número de casos analisados sobe para 13.916.407, correspondendo a 1048 anos 
simulados. Para realizar esta análise foram necessários 357 minutos. Os índices obtidos são: $\operatorname{LOLP}\left(\times 10^{2}\right)$ $=4,6682 ; \operatorname{EENS}(\mathrm{GWh} / \mathrm{a})=26,455 ; \operatorname{LOLF}($ oc./a) $=$ 155,12 e LOLD $(\mathrm{h})=2,6289$. Estes resultados comprovam o excelente desempenho da simulação Monte Carlo não-seqüencial proposta, tanto em termos da precisão dos índices estimados quanto em relação ao esforço computacional. Vale destacar que o speed up obtido ao se considerar esta última análise ficou igual a 54 vezes.

\section{CONCLUSÃO}

Este artigo apresenta uma nova metodologia para avaliar a confiabilidade de sistemas de potência considerando cargas variantes no tempo. A utilização de um modelo de Markov não-agregado com múltiplos níveis, para representar a carga, e de um novo processo para estimar a LOLF, denominado processo de transição de estado um passo à frente, traz para a simulação Monte Carlo não-seqüencial uma flexibilidade muito próxima daquelas apresentadas pelas técnicas seqüenciais. Os resultados obtidos utilizando o sistema teste MRTS e uma configuração do sistema Sul-Sudeste Brasileiro comprovam a eficácia desta nova metodologia.

\section{REFERÊNCIAS}

Anderberg, M.R., (1973). "Cluster Analysis for Applications", Academic Press, N.Y.

Breipohl, A.M., Lee, F.N., Zhai, D., Adapa, R., (1992). "A Gauss-Markov Load Model for Application in Risk Evaluation and Production Simulation", IEEE Trans. Power Syst., Vol. 7, No. 4, pp. 1493-1499.

EPRI, (1982). "Transmission system reliability models", Report EL-2526.

IEEE Reliability Test System Task Force of the Application of Probability Methods Subcommittee, (1979). "IEEE reliability test system", IEEE Trans. on PAS, 98, pp. 2047-2054.

Leite da Silva, A.M., Manso, L.A.F., Mello, J.C.O. and Billinton, R., (2000). "Pseudo-Chronological Simulation for Composite Reliability Analysis with Time Varying Loads", IEEE Trans. Power Syst., Vol. 15, No. 1, pp. 73-80.

Manso, L.A.F., Leite da Silva, A.M., and Mello, J.C.O., (1999). "Comparison of Alternative Methods for Evaluating Loss of Load Costs in Generation and Transmission System", Electric Power Systems Research, Vol. 50, pp. 107-114.
Mello, J.C.O., Pereira, M.V.F. and Leite da Silva, A.M., (1994). "Evaluation of reliability worth in composite system based on pseudo-sequential Monte Carlo simulation", IEEE Trans. Power Syst., 9, pp. 1318-1326.

Mello, J.C.O., Leite da Silva, A.M. and Pereira, M.V.F., (1997 a). "Efficient loss of load cost evaluation by combined pseudo-sequential and state transition simulation", IEE Proc. C, 141, pp. 147-154.

Mello, J.C.O., Pereira, M.V.F., Leite da Silva, A.M. and Melo, A.C.G., (1997 b). "Application of Chronological Load Modeling in Composite Reliability Worth Evaluation", Electric Power Systems Research, Vol. 40, pp. 167-174.

Melo, A.C.G., Pereira, M.V.F. and Leite da Silva, A.M., (1992). "Frequency and Duration Calculations in Composite Generation and Transmission Reliability Evaluation", IEEE Trans. Power Syst., Vol. 7, No. 2, pp. 469-476.

Melo, A.C.G., Pereira, M.V. and Leite da Silva, A.M., (1993). "A Conditional Probability Approach to the Calculation of Frequency and Duration Indices in Composite Reliability Evaluations", IEEE Trans. Power Syst., Vol. 8, No. 3, pp.1118-1125.

Pereira, M.V.F. and Balu, N.J., (1992). "Composite generation/ transmission reliability evaluation", Proceedings of IEEE, Vol. 80, No. 4, pp. 470-491.

Salvaderi, L., (1990). "Monte Carlo simulation techniques in reliability assessment of composite generation and transmission systems", IEEE Tutorial Course 90EH0311-1-PWR.

Sankarakrishnan, A. and Billinton, R., (1995). "Sequential Monte Carlo Simulation for Composite Power System Reliability Analysis with Time Varying Loads", IEEE Trans. Power Syst., Vol. 10, No. 3, pp. 1540-1545.

Singh, C., Pravin Chander, T. and Feng, J., (1993). "Convergence Characteristics of Two Monte Carlo Models for Reliability Evaluation of Interconnected Power Systems", Electric Power Systems Research, Vol. 28, pp. 1-8.

Wenyuan, L., Billinton, R., (1991). "Effects of bus load uncertainty and correlation in composite system adequacy evaluation", IEEE Trans. Power Syst., Vol. 6, No. 4, pp. 1522-1529. 